\title{
Social Oocyte Freezing: A Boon for Aging Females
}

\author{
Deepa Talreja ${ }^{1}$, Nandita Palshetkar ${ }^{2}$, Hrishikesh Pai ${ }^{3}$
}

\begin{abstract}
Reproductive aging plays a very important role in female fertility. The age-related decline in fertility can be attributed to a decrease in number as well as poor quality of oocytes. The trend to delay childbearing confronts women with difficulty in conception. Oocyte freezing at a younger age provides an option to freeze the eggs before the biological clocks start ticking. It allows women to freeze their eggs at the peak of fertility and create embryos at the later stage when they find a suitable partner or when they are ready to pursue the family. Introduction of new "vitrification" technique has made the success rates for actual conception more reliable than the earlier method of slow freezing. Due to improved results with the vitrification technique, and various studies reporting improved pregnancy rates, oocyte freezing is now no longer considered to be experimental. Oocyte freezing with egg banking has now wider application in fertility services.
\end{abstract}

Keywords: Fertility preservation, Oocyte cryopreservation, Vitrification.

International Journal of Infertility and Fetal Medicine (2018): 10.5005/jp-journals-10016-1169

\section{INTRODUCTION}

Reproductive aging plays an important part in female fertility as pregnancy rate decreases exponentially after the age of 37 and this decline is further accelerated after 40 years. ${ }^{1}$ This age-related decline can be attributed to a decrease in number as well as poor quality of oocytes. The trend to delay childbearing in modern societies confronts women with difficulty in conception.

Women at an advanced age have increased chances of miscarriage if they can get pregnant at all. Donor eggs are the option in such cases, but for many women, it is not acceptable because of ethical and social concerns. An emerging preventive solution for women is to freeze their oocytes at the younger age for future use, so that they can create a form of insurance against age-related decline in fertility.

Social oocyte freezing has become very popular in the past 5 years, due to the improved outcome of new technologies of assisted reproductive techniques. It is the option to freeze the eggs before the biological clock starts ticking. It allows women to freeze their eggs at the peak of fertility and create embryos at a later stage when they find a suitable partner or when they are ready to pursue the family.

Cryopreservation of oocytes is a relatively recent development in the field of assisted reproductive technique (ART). The first pregnancy after oocyte cryopreservation was reported in 1986 by Chen. ${ }^{2}$ It had a low success rate in the past; however, the introduction of the new "vitrification" technique has made the success rates for actual conception more reliable than the earlier method of slow freezing.

Considering its success rate on the basis of the available evidence, the American Society of Reproductive Medicine has recently declared that oocyte cryopreservation should no longer be considered experimental for medical indications, outlying elective oocyte cryopreservation. ${ }^{3}$ Since then, oocyte freezing and egg banking have been proposed for various new horizons of indications.

\section{Case History}

The patient was a 33-year-old unmarried female, who visited our outpatient department (OPD) in 2007 with a wish of freezing her eggs, as she wanted to preserve her fertility till she will get
${ }^{1}$ IVF Department, Lilavati Hospital and Research Centre, Mumbai, Maharashtra, India.

${ }^{2,3}$ Department of OBGY, Bloom IVF, Navi Mumbai, Maharashtra, India

Corresponding Author: Deepa Talreja, IVF Department, Lilavati Hospital and Research Centre, Mumbai, Maharashtra, India, Phone: +91 7506987141, e-mail: drdeepa30@gmail.com

How to cite this article: Talreja D, Palshetkar N, et al. Social Oocyte Freezing: A Boon for Aging Females. Int J Infertil Fetal Med 2018;9(3):41-44.

Source of support: Nil

Conflict of interest: None

married and find a right partner. The pre-art evaluation was done including history, examination, and investigations needed. Flexible antagonist protocol was used for ovarian stimulation, starting from the second day of the menstrual cycle using highly purified human menopausal gonadotropin (HMG). Monitoring was done by serial ultrasonography and serum hormonal levels. Antagonist (inj. Cetrorelix $0.25 \mathrm{mg} \mathrm{S/C}$ daily) was started when leading follicle reached $14 \mathrm{~mm}$ in diameter. Injection HCG 10,000 IU given intramuscularly to trigger final oocyte maturation. Transvaginal ultrasound-guided oocyte retrieval was done after 34 hours of injection HCG under anesthesia.

Oocyte cryopreservation was initiated 2 hours postretrieval, after denuding the cumulus cells chemically (with hyaluronidase) and mechanically and confirming metaphase II oocytes. The vitrification technique using the Cryotop method was used for oocyte freezing.

Vitrification of oocytes was done by the minimum volume cooling (MVC) method. Briefly, the equilibration of oocytes was done in the equilibration medium (basal medium with $7.5 \%(\mathrm{v} / \mathrm{v})$ ethylene glycol and 7.5\% (v/v) dimethylsulfoxide, i.e., DMSO) at room temperature for 12-15 minutes, depending on the recovery of oocytes. Then oocytes were transferred into the vitrification medium (basal medium with 15\% (v/v) ethylene glycol, 15\% (v/v) DMSO, and $0.5 \mathrm{M}$ sucrose) at room temperature for 45-60 seconds. The cryoprotectants-treated oocytes were loaded onto a fine polypropylene strip (Cryotop; Kitazato Bio Pharma Co.). Finally, 
polypropylene strips carrying oocytes were immediately submerged into liquid nitrogen. A total of nine MIl oocytes were frozen. Figures 1 to 5 describe the procedure of vitrification and final loading of oocytes.

The patient underwent the second cycle after a period of 2 months and seven MII oocytes were frozen. A total of 16 mature oocytes were frozen from two oocyte retrievals.

In 2015, she visited our OPD, as she got married and decided to plan for conception (she was 41 years old at that time). After initial evaluation for both male and female partners, she decided to start with intrauterine insemination (IUI). She underwent 2 cycles of IUI but failed to conceive. During this period, she was also diagnosed to have endometriosis, a disease which further affects the fertility. So, she has been advised to go ahead with in vitro fertilization/ intracytoplasmic sperm injection (IVF/ICSI) with her frozen eggs.

The endometrial preparation was done by the hormone replacement method, with injection $\mathrm{GnRH}$ agonist depot for suppression of ovarian function. After confirming down-regulation, the preparation of endometrium was started with incremental doses of estradiol valerate $2 \mathrm{mg}$, with addition of vaginal micronized progesterone $200 \mathrm{mg}$ twice a day, from the day of thawing (or warming).

For the warming process, the polypropylene strips with vitrified oocytes were removed from the liquid nitrogen and were immersed

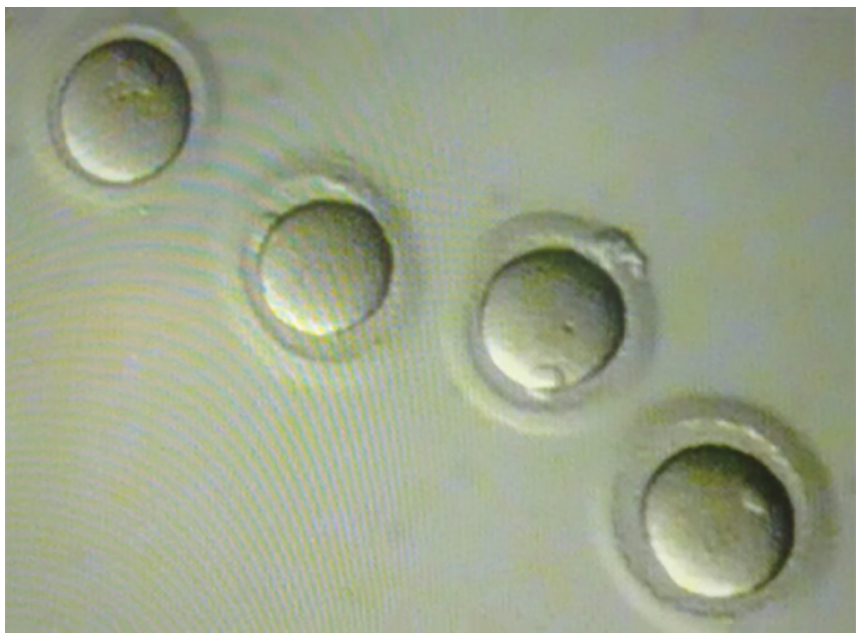

Fig. 1: Mature oocytes after denuding

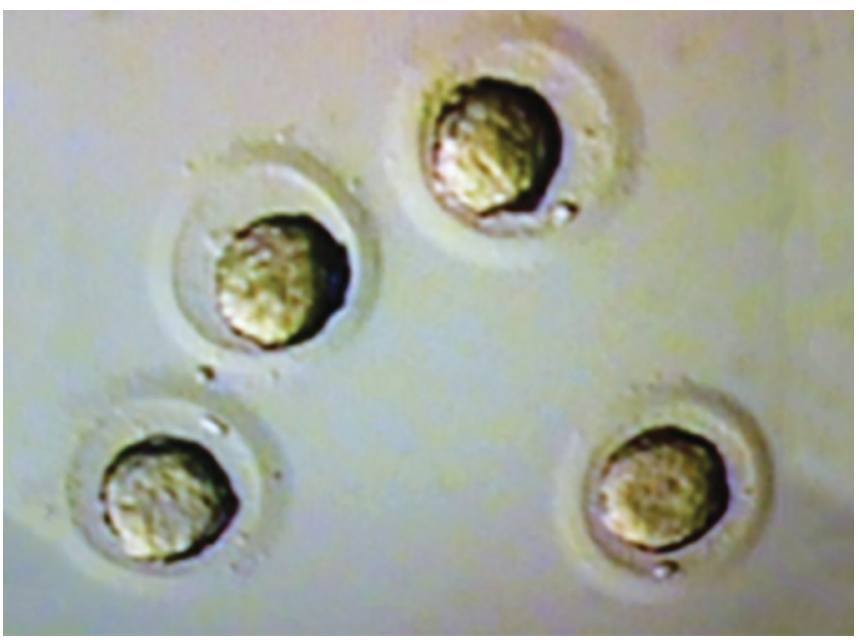

Fig. 3: Shrinkage of oocytes 6 minute after transfer to equilibration solution (ES) directly into $0.8 \mathrm{~mL}$ of thawing solution (hydroxyethyl piperazine ethanesulfonic acid [HEPES]-buffered medium with $20 \%$ (v/v) serum protein substitute and $1.0 \mathrm{M}$ sucrose) at $37^{\circ} \mathrm{C}$ for 1 minute. Oocytes were then picked up and transferred into $0.3 \mathrm{~mL}$ of the dilution solution (HEPES-buffered embryo culture medium with $20 \%(\mathrm{v} / \mathrm{v})$ SPS and $0.5 \mathrm{M}$ sucrose) for 3 minutes at room temperature. Oocytes were washed in $0.3 \mathrm{~mL}$ washing solutions, i.e., washing solutions 1 and 2 (HEPES-buffered embryo culture medium with $20 \%$ (v/v) SPS) for 5 minutes each at room temperature subsequently.

A survival check was carried out under the inverted microscope. Oocytes with intact zona, clear perivitelline space, and visible polar body were considered as normal and were kept in the culture media at $37^{\circ} \mathrm{C}$ and $5 \% \mathrm{CO}_{2}$ for at least 2 hours before $\mathrm{CSI}$. Subsequently, partner's sperm preparation was done by density gradient centrifugation, and fertilization using intracytoplasmic sperm injection (ICSI) was performed.

Fertilized oocytes were kept in cleavage media, in a trigas incubator for further culture until pronuclear check 16-18 hours later. Embryos were evaluated on day 3, and assessed for development and quality. Good quality day-3 embryos were defined as $6-8$ cells and $<10 \%$ fragmentation.

A total of nine embryos were formed. Two good quality embryos were transferred, but she failed to conceive. She decided

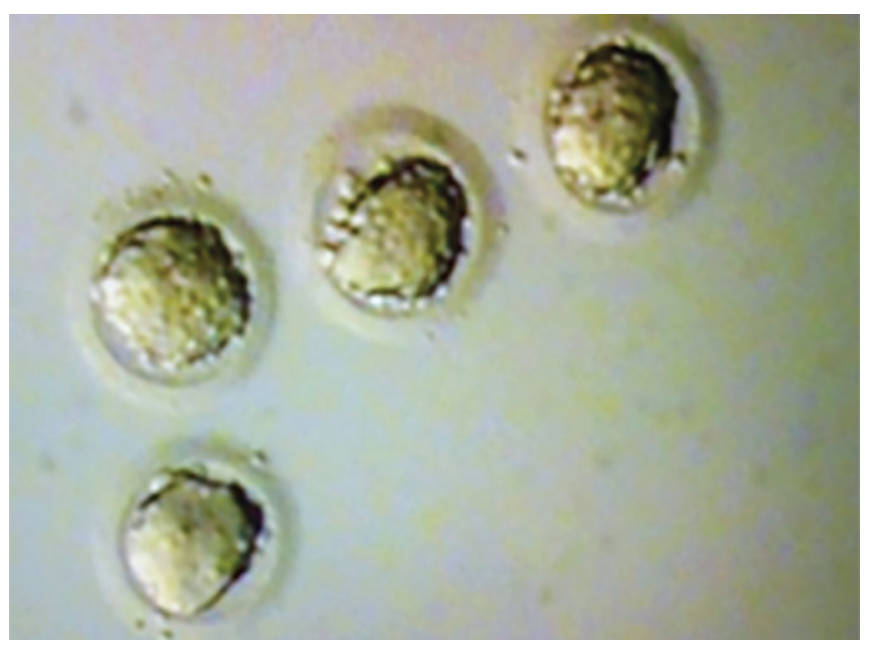

Fig. 2: Shrinkage of oocytes 1 minute after transfer to equilibration solution (ES)

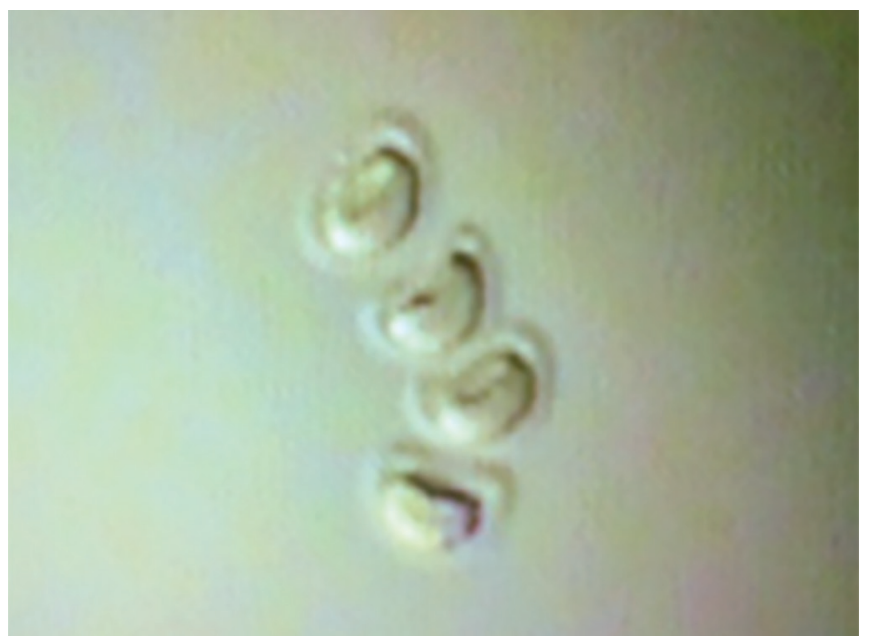

Fig. 4: Shrinkage of oocytes (almost $50 \%$ of its volume) again in vitrification solution (VS) 


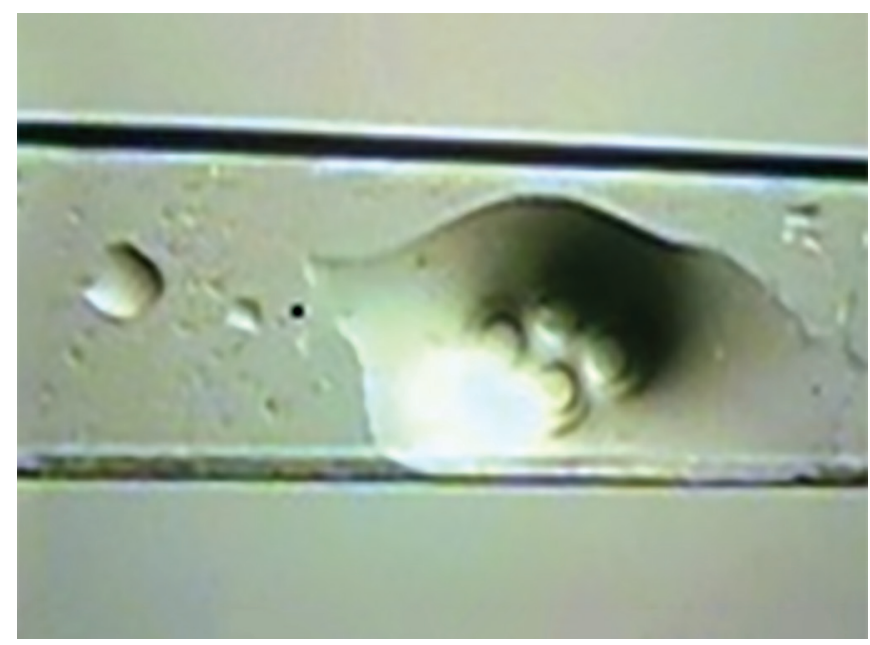

Fig. 5: In the above stage oocytes are loaded on the Cryotop device

to go with the second frozen embryo transfer. A similar hormonal method was used for endometrial preparation. This time three good quality embryos were transferred. A pregnancy test done after 14 days came positive. Her antenatal period was uneventful. She underwent full-term elective lower segment cesarean section (LSCS) in January 2016 and delivered a healthy female child with a birth weight of $3.5 \mathrm{~kg}$ (at the age of 42 years). A routine follow-up at 3 months and 6 months showed normal development for age.

\section{Discussion}

In the present case report, we demonstrated that oocyte freezing at a younger age provides an option for women to preserve their fertility. Although the author has performed oocyte cryopreservation for social reasons in many cases, this case was particularly discussed as it highlights the current trend of delaying pregnancy. This case also provides a systematic methodology of oocyte freezing and successful outcome. Oocyte freezing with egg banking has now wider application in fertility services, mainly due to improved results with the vitrification technique.

There are several indications for an efficient oocyte freezing and cryostorage program including women with malignant/ premalignant conditions to preserve their reproductive capacity before undergoing potentially sterilizing cancer treatment, ${ }^{4,5}$ government restrictions on embryo freezing due to ethical reasons, ${ }^{6}$ women at risk of premature ovarian failure, and for other practical reasons, such as unexpected semen collection problems on the day of ovum pickup.

Social oocyte freezing has gained wider popularity, as it provides a technical solution to a number of various problems women face due to the extended education and waiting to establish their household and career. ${ }^{7}$

Cryopreservation refers to the cooling of cells and tissues to sub-zero temperatures to stop all biologic activity and preserve them for future use. The most challenging step in cryopreservation probably is to maintain plasma and membrane integrity by preventing ice crystal formation. To prevent this injury, various permeating and non-permeating cryoprotectants are used at different concentrations. However, some of these cryoprotectants themselves are toxic at higher concentrations. So, to avoid these problems, an intrinsic balance is needed between the concentrations and the exposure time of cryoprotectants. ${ }^{8}$
Two techniques commonly used for cryopreservation are slow freezing and vitrification. The slow-freezing method is a freezing method, where extracellular ice formation drives cellular dehydration through an equilibrium process and uses a low concentration of cryoprotectants for an extended time before the actual freezing occurs. Vitrification is nonequilibrium cooling which utilizes very high concentrations of cryoprotectants that solidify without forming ice crystals.

Vitrification has gained popularity in freezing the oocytes due to its superiority to the slow-freezing method, in the form of better oocyte survival rate, fertilization, and embryonic development in vitro.

Cochrane review including two randomized controlled trials (RCTs) with 106 participants concluded that the vitrification technique was associated with an increased clinical pregnancy rate compared to the slow-freezing method in women undergoing assisted reproduction (risk ratio [RR] 3.86, 95\% Cl 1.63-9.11, $p=0.002$ ). ${ }^{9}$

Several studies have been conducted to evaluate the outcome of oocyte cryopreservation. In a prospective randomized study, Cobo et al. analyzed the outcome of oocyte vitrification using the Cryotop method and concluded that there was no difference in fertilization rates, day 2 cleavage rates, blastocyst formation for vitrified and fresh oocytes, and noted a survival rate of $96.7 \%$. The authors conclude that the Cryotop method preserves the potential of vitrified oocytes to fertilize and further development. They recommended the possible use of this technique for egg donation program, as well as for establishing oocyte banking. ${ }^{10}$

In a systematic review by American Society of Reproductive Medicine (ASRM) committee outcomes of four randomized controlled trials were compared for cryopreserved and fresh oocytes in IVF/ICSI cycles. Overall, oocyte survival after vitrification and warming ranged between 90 and $97 \%$, fertilization rates were between 71 and $79 \%$, implantation rates were 17-41\%, and clinical pregnancy rates per transfer ranged from 36 to $61 \% .^{3}$

Various concerns have been expressed with oocyte freezing. One of the main concerns was the damage to the meiotic spindle as an effect of freezing, leading to chromosomal or other cellular abnormalities in embryos derived from frozen eggs. However, studies are reassuring in this regard. A study by Cobo et al. showed no increase in numerical chromosomal abnormalities in embryos derived from oocytes slow-frozen compared with non-frozen controls. ${ }^{11}$

Despite the technical expertise of freeze/thaw process, the most important determining factor is the age of women at oocyte freezing. The ideal age for oocyte freezing is 30-35 years, as women in their late 20s still have the time to find a partner and oocytes from women over 35 years will produce fewer pregnancies in comparison to women under 35 years due to age-related decline in oocyte quality.

Another important factor to be concerned is the number of oocytes to be frozen. An observational longitudinal cohort multicenter study conducted by Rienzi et al. concluded that more than eight vitrified oocytes are required to improve the outcome and delivery rates. ${ }^{12} \mathrm{~A}$ latest study also supports the concept that at least 8-10 metaphase ll oocytes are necessary to achieve reasonable success. Numbers should be individualized in women $>36$ years old (Cobo et al.). ${ }^{13}$

Another factor determining a successful conception is the reproductive potential of a female, simply defined as "Ovarian reserve." Although a decrease in ovarian reserve is often noted in women in their mid to late 30s, but it may affect younger women 
as well. So, the identification of ovarian reserve at an earlier stage would help women who wish to delay childbearing to assist in making an informed decision.

\section{Conclusion}

The current case report supports the concept that oocyte cryopreservation can be performed successfully, providing the basis to apply it for various indications like social oocyte freezing. Social oocyte freezing has also recently been defined as a sort of "reproductive insurance" against age-related infertility, which empowers women by giving them a way to preserve oocytes against the threat of age or disease. Although several studies have also highlighted the importance of social oocyte freezing, the aim of the above case report is to increase awareness. It is merely a case report and the author recommends that further studies will be needed to determine the efficacy and safety of this technique. The author also emphasizes that women should be properly informed about the present uncertainties related to the efficiency and safety of future reproductive use of their frozen oocytes.

\section{References}

1. Practice Committee of American Society for Reproductive Medicine. Age related fertility decline: a committee opinion. Fertil Sterile 2008;90(5 Suppl):S154-S155. DOI: 10.1016/j.fertnstert. 2008.08.006.

2. Chen C. Pregnancy after human oocyte cryopreservation. Lancet 1986;1:884-886. DOI: 10.1016/S0140-6736(86)90989-X.

3. Practice Committee of American Society for Reproductive Medicine; Society for Assisted Reproductive Technology. Mature Oocyte
Cryopreservation: a guideline. Fertil Steril 2013;99:37-43. DOI 10.1016/j.fertnstert.2012.09.028.

4. Rao GD, Chian RC, et al. Fertility preservation in women undergoing cancer treatment. Lancet 2004;363:1829-1830. DOI: 10.1016/S01406736(04)16320-4.

5. Del Mastro L, Venturini M. Fertility preservation strategies for breast cancer patients. J Clin Oncol 2006;24:4220-4221. DOI: 10.1200/ JCO.2006.07.4310.

6. Boggio A. Italy enacts new law on medically assisted reproduction. Hum Reprod 2005;20:1153-1157. DOI: 10.1093/humrep/deh871.

7. Waldby C. 'Banking time': egg freezing and the negotiation of future fertility. Cult Health Sex 2015;17(4):470-482. DOI: 10.1080/13691058.2014.951881.

8. Nagy ZP, Chang CC, et al. Clinical evaluation of the efficiency of an oocyte donation program using egg cryo-banking. Fertil Steril 2009 Aug;92(2):520-526. DOI: 10.1016/j.fertnstert.2008.06.005.

9. Glujovsky D, Riestra B, et al. Vitrification vs slow freezing for women undergoing oocyte cryopreservation. Cochrane Database Syst Rev 2014 Sep 5;9:CD010047. DOI: 10.1002/14651858.CD010047.pub2.

10. Cobo A, Kuwayama M, et al. Comparison of concomitant outcome achieved with fresh and cryopreserved donor oocytes vitrified by the Cryotop method. Fertil Steril 2008 Jun;89(6):1657-1664. DOI: 10.1016/j.fertnstert.2007.05.050.

11. Cobo A, Remohi J, et al. Oocyte cryopreservation for donor egg banking. Reprod Biomed Online 2011 Sep;23(3):341-346. DOI 10.1016/j.rbmo.2011.05.014.

12. Rienzi $\mathrm{L}, \mathrm{Cobo} A$, et al. Consistent and predictable delivery rates after oocyte vitrification: an observational longitudinal cohort multicentric study. Hum Reprod 2012 Jun;27(6):1606-1612. DOI: 10.1093/humrep/ des088.

13. Cobo A, García-Velasco JA, et al. Oocyte vitrification as an efficient option for elective fertility preservation. Fertil Steril 2016 Mar;105(3):755-764. DOI: 10.1016/j.fertnstert.2015.11.027. 